\title{
DRAFT: SINGLE AND MULTIPLE CIRCUMFERENTIAL CASING GROOVE FOR STALL MARGIN IMPROVEMENT IN A TRANSONIC AXIAL COMPRESSOR
}

\author{
A. F. Mustaffa* and V. Kanjirakkad \\ Thermo-Fluid Mechanics Research Centre \\ School of Engineering \& Informatics \\ University of Sussex \\ Falmer, UK BN19QH \\ Email: A.F.Mustaffa@sussex.ac.uk
}

\begin{abstract}
The stability limit of a tip-stalling axial compressor is sensitive to the magnitude of the near casing blockage. In transonic compressors, the presence of the passage shock could be a major cause for the blockage. Identification and elimination of this blockage could be key to improving the stability limit of the compressor. In this paper, using numerical simulation, the near casing blockage within the transonic rotor, NASA Rotor 37, is quantified using a blockage parameter. For a smooth casing, the blockage at conditions near stall has been found to be maximum at about $20 \%$ of the tip axial chord downstream of the tip leading edge. This maximum blockage location is found to be consistent with the location of the passage shock-tip leakage vortex interaction. A datum single casing groove design that minimises the peak blockage is found through an optimisation approach. The stall margin improvement of the datum casing groove is about $0.6 \%$ with negligible efficiency penalty. Furthermore, the location of the casing groove is varied upstream and downstream of the datum location. It is shown that the stability limit of the compressor is best improved when the blockage is reduced upstream of the peak blockage location. The paper also discusses the prospects of a multi-groove casing configuration.
\end{abstract}

Keywords: Stall margin improvement, axial compressor, casing treatment

\section{NOMENCLATURE}

$\begin{array}{ll}c_{a x, t} & \text { Tip axial chord } \\ H & \text { Groove height } \\ i & i^{t h} \text { grid cell } \\ \text { LE } & \text { Leading edge } \\ \mathrm{m} & \text { Mass flow rate }\end{array}$

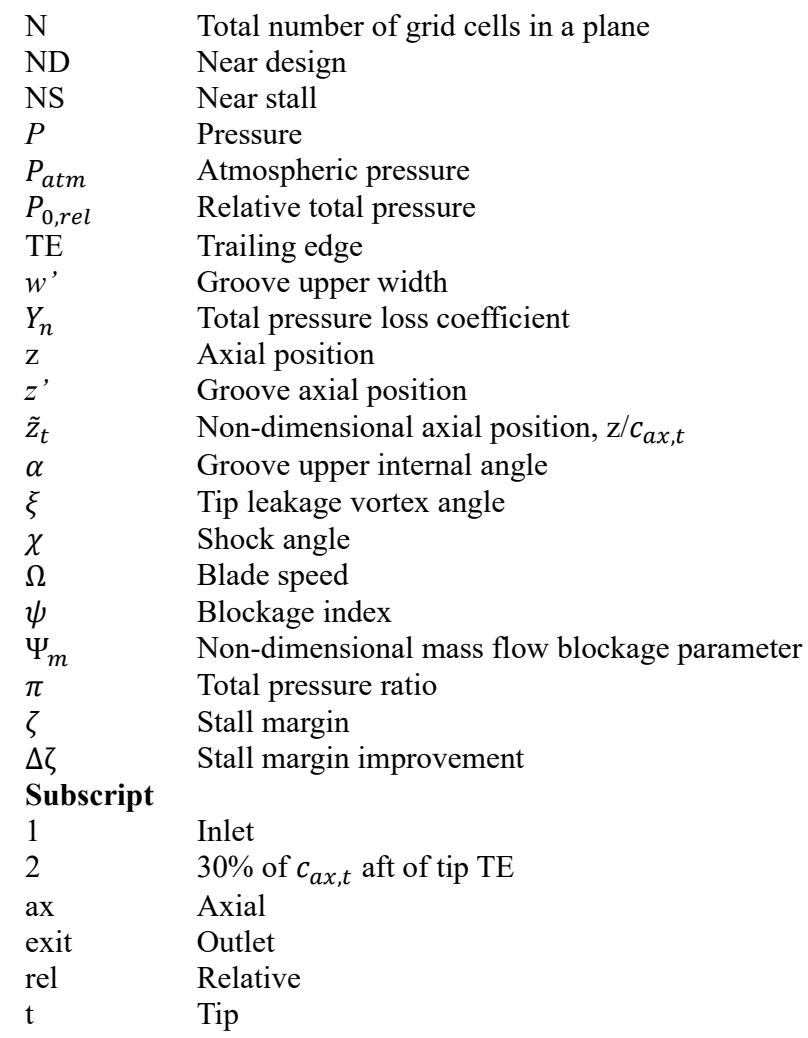

\section{INTRODUCTION}

The stable operating range of tip-critical axial compressors is limited by the magnitude of the near casing blockage. For a highspeed transonic compressor, Suder and Celestina [1] have shown that the passage shock-tip leakage vortex (TLV) interaction is 
responsible for the near casing blockage. The near casing blockage is quantified to be two or three times higher than the core flow region as the compressor approaches stall. Recently, in another high-speed compressor rig, Brandstetter et al. [2] used optical measurements to investigate the aerodynamic phenomenon in the near casing region to understand the mechanism of rotating stall. At conditions near-stall, it was observed that a large blockage region exists in the blade passage due to the breakdown of the tip leakage vortex. Subsequently, the blockage region causes the flow to 'spill forward' into the adjacent passage. The flow at the leading edge of the adjacent blade separates as a result of this 'spill forward' effect. The separation is shed into the blade passage as radial vortices. Radial vortices are already known to be one of the mechanism responsible for the inception of spike stall in low-speed subsonic compressors as reported in $[3,4]$.

In order to alleviate the blockage associated to the tip region aerodynamics, casing treatment such as circumferential casing grooves has been used to improve the stall margin of high-speed axial compressors. The concept of casing groove for stall margin improvement (SMI) has emerged since the early 1970's. This is long before the aforementioned explanation for the casing blockage growth at conditions near stall are known. Bailey [5] tested multiple casing groove configurations on a single stage transonic axial compressor. Three different casing groove depths were tested with the number of grooves and location varied using aluminium inserts. At design speed, the deepest groove showed the best performance as compared to the shallow ones. The highest SMI was achieved when only five of the nine deep grooves located at about the tip mid-chord were opened.

Muller et al. [6] combined experimental and steady-state numerical simulations to explain the physical effect of the grooves on the tip region flow. Four casing groove configurations on a single stage transonic axial compressor at different rotor speeds were studied. The configurations consist of deep and shallow grooves with multiple deep grooves covering the rotor tip chord. At design speed, the best SMI was obtained when deep grooves that covered almost the whole length of the tip chord were used. Near casing Mach number contours obtained from their numerical simulation showed that the near casing blockage area was reduced by the grooves. The reduction of blockage area is caused by the interaction between the leakage flow and the flow into and out of the groove. This prevented the 'spill forward' effect thus delaying the onset of stall.

Sakuma et al. [7] studied the effect of the location and depth of a single casing groove on a transonic isolated axial compressor rotor using numerical simulations. The location of the groove was varied axially over the tip. The groove with the greatest SMI was found when the deep groove was located at $20 \%$ of $c_{a x, t}$ aft of the tip leading edge. It was explained that the groove at this location causes a large reduction of the tip leakage flow momentum. The reduction of the leakage momentum led to the deflection of the TLV and hence resulted in shifting the location of the blockage region.

Chen et al. [8] numerically analysed the effect of multiple casing grooves on the tip axial flow momentum in a single stage transonic compressor. It was found that the flow into and out of the grooves provide a positive net axial momentum that reduces the backward momentum of the tip leakage flow. The greatest contribution came from the first four grooves located aft of the tip leading edge. The reduction in backward momentum in return results in the improvement of the stall margin. This reduction of reverse axial momentum in other words, can be interpreted as the reduction of blockage. Ross et al. [9] tested seven casing groove configurations on the same transonic compressor. This study was conducted to investigate the effects of casing groove number and location on the SMI of the compressor. By performing an analysis of the tip region smooth casing axial momentum flux density, a linear relationship between SMI and the casing groove location was found. Adding each of the individual grooves together provided a linear increase to the SMI.

From the literature, it is clear that the SMI due to casing grooves can be linked to the reduction of the near-casing blockage. However, these findings are often based on a 'blackbox' approach. This is rather expensive as it requires all possible casing groove design and configurations to be tested first, in order to find the best possible case or configuration. In addition, a 'black-box' approach is a disadvantage to a designer as the physical significance of each design cannot be clearly explained. Therefore, this paper aims to show that the design of casing grooves can be done through a physics-based approach. First, the tip region blockage of a smooth casing is quantified to find where the blockage is maximum. Based on this information, a single groove design is obtained through a design optimisation process. Next, the location of the optimised single casing groove design is varied across the maximum blockage region to study the sensitivity of the groove location to the SMI. Lastly, the prospect of a multi-groove configuration is investigated by combining the individual grooves.

\section{NUMERICAL METHOD}

\section{Numerical domain and grid}

The present study is performed on an isolated transonic axial compressor, NASA Rotor 37. The meridional cut-view of the numerical domain is shown in Fig. 1. The design variables of the casing groove are shown in the inset of Fig. 1. Details regarding the aerodynamic design specification of the rotor blade

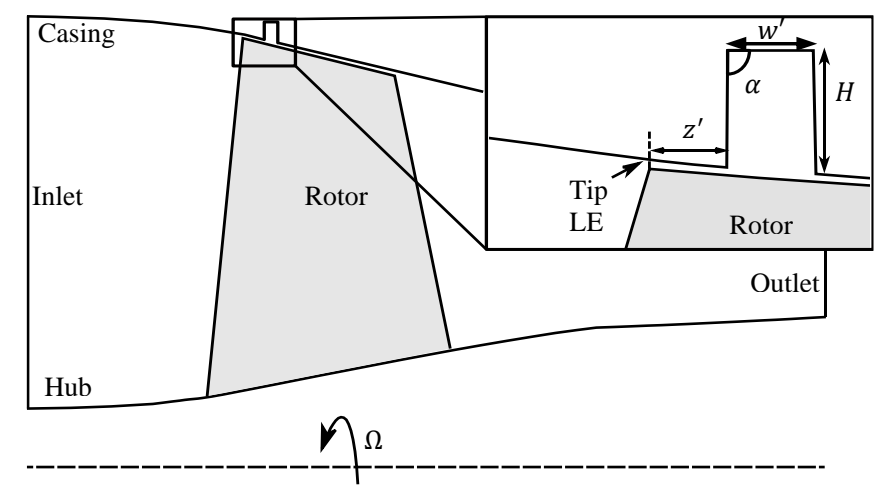

FIGURE 1. Meridional cut-view of NASA Rotor 37 and groove design parameters 
TABLE 1. Design specifications of NASA Rotor 37

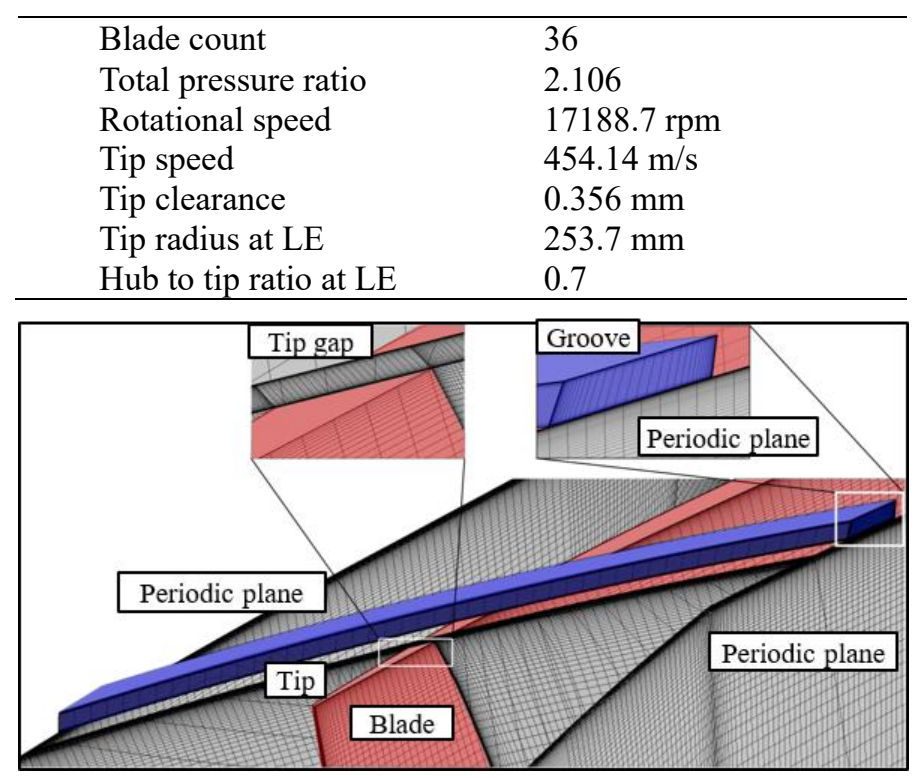

FIGURE 2. The grid near the casing and groove

row are shown in Tab. 1. For more details about Rotor 37 test case, the reader is referred to $[10,11]$.

Figure 2 shows the numerical grid points generated for a single blade passage and groove domain. The blade passage has approximately a total of 4 million grid points with 30 radial grid points within the tip gap itself. The groove domain has about 180,000 grid points and is generated so that the ends of the groove match the periodic plane of the blade passage domain. By having a matching interface between the groove and blade passage domain, the entire domain can be treated as a single domain in the rotating frame of reference. The grid between the blade passage and groove are connected via a General Grid Interface (GGI). One of the advantages of a GGI connection is that it allows for a non-overlap boundary condition [12]. The casing surface that do not overlap with the groove is treated as a no-slip, adiabatic and impermeable wall. As a result, any parametric study on the groove will only require modifications to the groove domain without any change to the blade passage grid.

\section{Boundary conditions}

Steady state simulations are performed by solving the 3D, compressible and adiabatic RANS equations using a commercial code, ANSYS CFX. A high order resolution scheme was set to discretise the conservative equations. A standard k-epsilon turbulence model is used for closing the RANS equations. At the inlet, a total pressure and total temperature profile is prescribed using the measured inlet conditions as found in [13]. Since the entire domain is solved in the rotating frame, counter-rotating wall condition is imposed to mimic stationary walls. The hub walls are set to fully rotating to re-create conditions that cause a hub total pressure deficit as discussed in [11]. The outlet static pressure is varied from a value of $P_{\text {exit }} / P_{\text {atm }}=1.05$ (choking point) until the solver fails to produce a converged solution. At this point, the compressor is said to have reached numerical stall. The numerical stall point is determined iteratively by increasing $P_{\text {exit }}$ until it fails to satisfy the following convergence criteria:

1. The calculations are run for a minimum of 2000 iterations.

2. Coefficient of variation (CV) of the inlet mass flow rate value must not exceed 0.001 for the last 200 iterations. (CV is the ratio between the standard deviation to the mean.)

3. Residuals for mass, momentum and energy for the last 1000 iterations behave normally.

The increment step-size of $P_{\text {exit }}$ follows the rule of a 'bisection method' and is updated until the step-size reaches 5 Pa.

\section{SMOOTH CASING RESULTS AND DISCUSSION}

The smooth casing is simulated at design $(100 \%)$ and part speed $(60 \%)$. At part-speed, the flow in the blade passage is reported to be free from passage shock [1]. The tip gap height increase and changes to blade twist at part-speed as reported in [1] are not modelled for this study. Therefore, the result of the part-speed simulation will not be compared to the actual test result and is only intended for comparing the near-casing blockage due to the influence of the passage shock. Figure 3 shows the total pressure ratio, $\pi$, of the smooth casing axial compressor along the design and part-speed lines. The calculated $\pi$ of the design speed is within a $2 \%$ error margin of the experiment results. Operating points 4 and 8 in Fig. 3a represents the near design (ND) and near stall (NS) cases from the measurements. Operating point 10 is the last stable operating point found using the procedure mentioned in the previous section.

The outlet radial distribution of $\pi$ and the adiabatic efficiency, $\eta$, for operating points 4 and 8 as shown in Fig. 4 suggests a good agreement with the trend of the measured

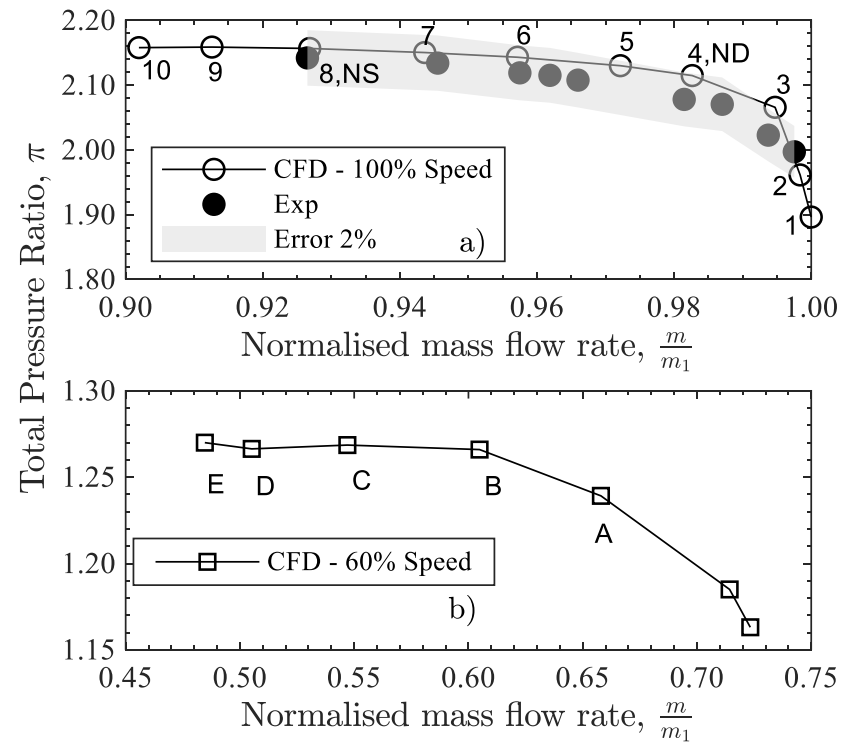

FIGURE 3. Performance curve for a) design speed and b) part-speed 

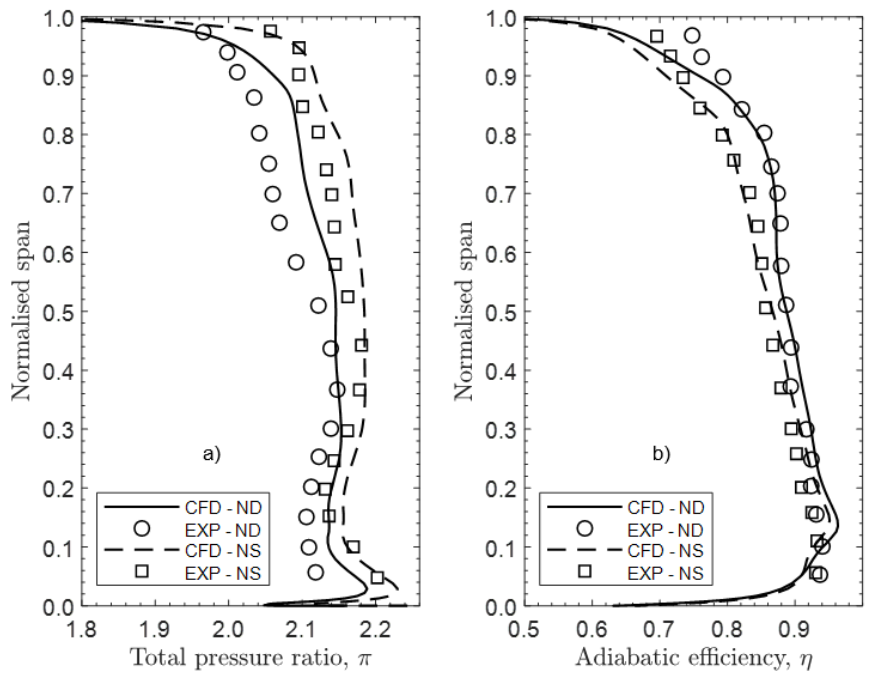

FIGURE 4. Outlet radial distribution of $\pi$ and $\eta$ for operating a) point 4 and b) point 8

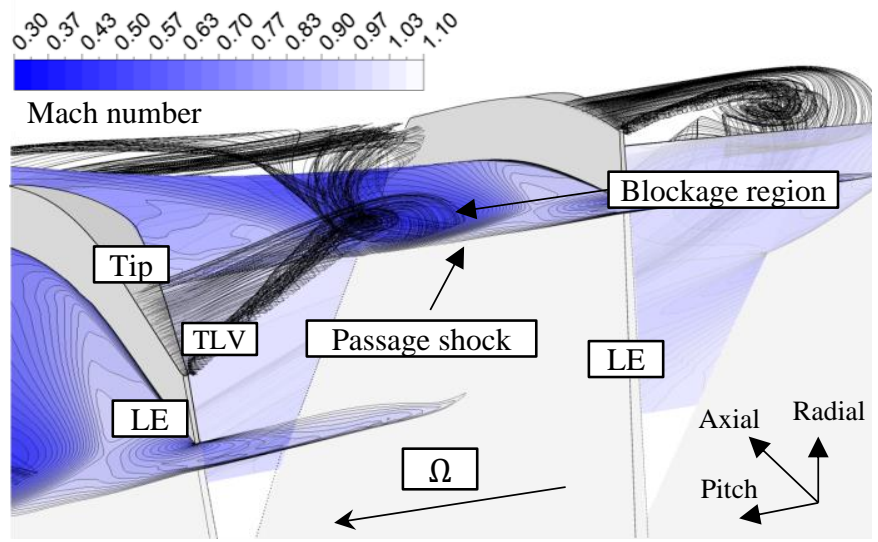

FIGURE 5. Mach number contour at $95 \%$ span and tip leakage vortex streamlines

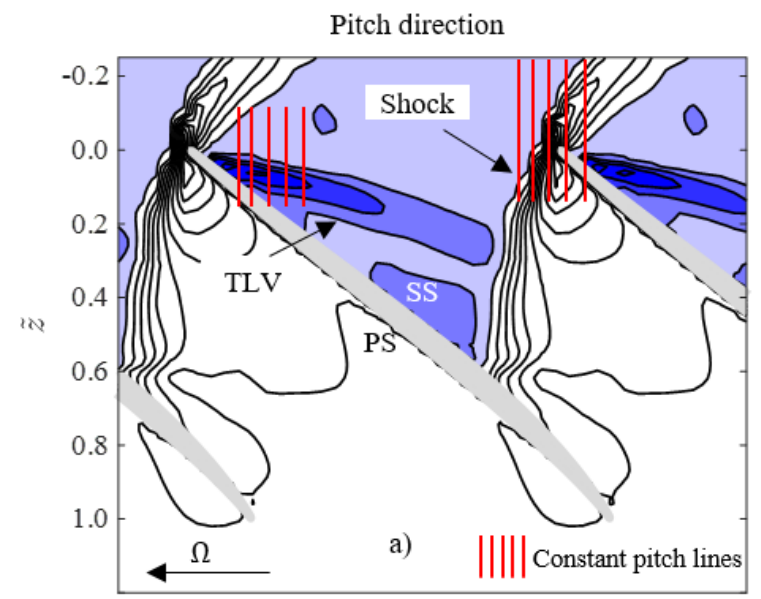

results. The quantities $\pi$ and $\eta$ are calculated using the same method found in [10]. The trend of $\eta$ near the endwalls shows a slight mismatch due to assumption of adiabatic conditions. It has been shown by Bruna el al. [14] that a better match at the endwalls can be obtained when using an isothermal boundary condition. The above authors assumed that the endwalls have the same temperature as the inlet total temperature since former was not measured. It was shown that the $\eta$ difference at the endwall between isothermal and adiabatic conditions was about $1 \%$ for the NS case. In reality, the endwall temperature would not be an isothermal condition, hence, it is suffice to show that the endwall $\eta$ distribution can better be simulated with real heat transfer effects.

\section{Shock-Tip leakage vortex (TLV) interaction}

Figure 5 shows the Mach number distribution at $95 \%$ span for operating point 8 . A relatively low Mach number region can be seen downstream of the shock-TLV interaction location. The TLV can be identified by the roll-up of the leakage flow that originates from the tip leading edge (LE). The interaction with the shock causes the TLV to disintegrate which may suggest a possible vortex breakdown although a 'bubble'-type breakdown as found by [15] has not been successfully captured. The relationship between the shock-TLV interaction and the blockage can be shown by first finding the location of this interaction. The location of the interaction can be approximated by finding the intersection point between the lines that are along the trajectory of the shock and TLV.

As shown in Fig. 6, the shock and TLV can be identified by a sudden pressure rise (bunching of contour lines) and the minimum pressure region, respectively. The trajectory line of the shock and TLV is found by extracting pressure profiles along several constant pitch lines as shown in Fig. 6. For the TLV trajectory line, the axial location of the minimum pressure for each extracted pressure profiles are found through interpolation.
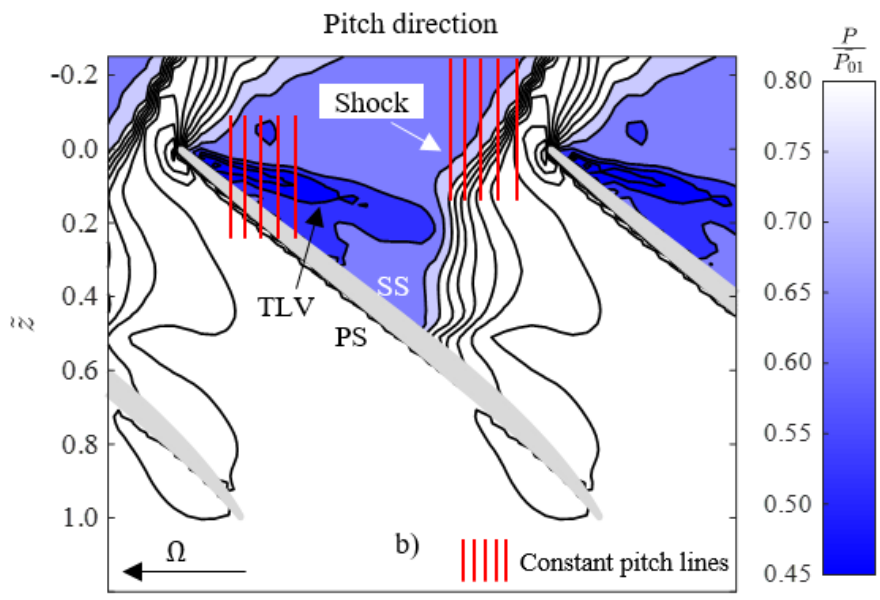

FIGURE 6. Casing static pressure for operating a) point 4 and b) point 8 


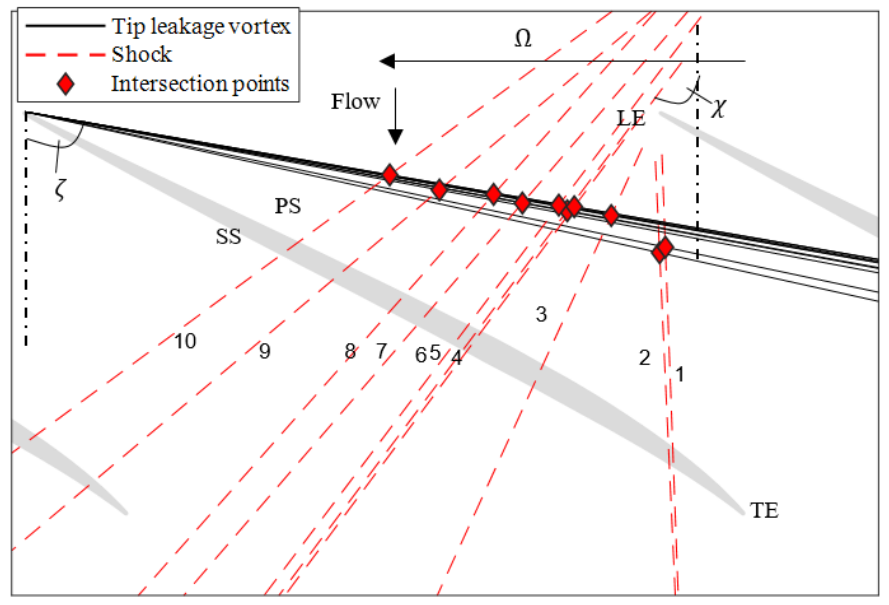

FIGURE 7. Shock and TLV trajectory line for all operating points
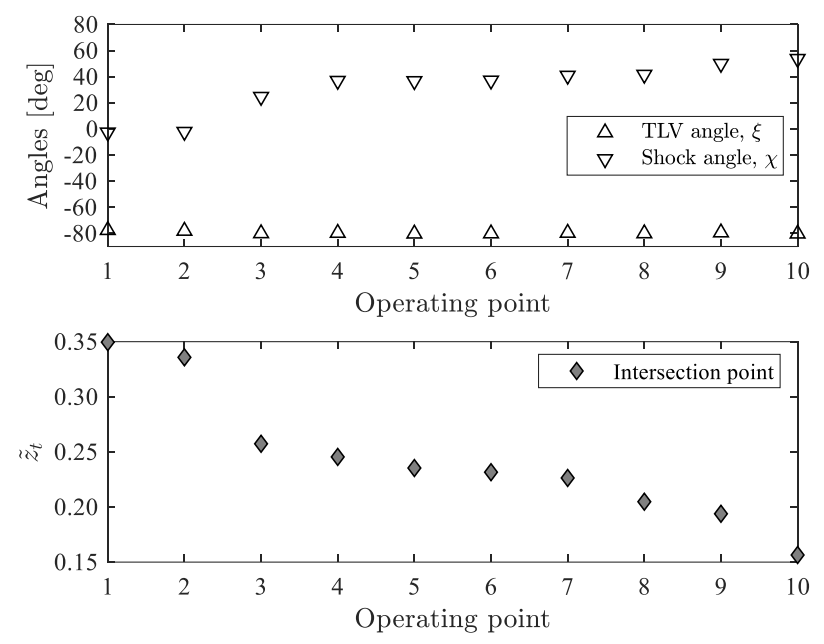

FIGURE 8. a) Shock and TLV angles and b) axial location of the shockTLV intersection

Since, the pitch and axial coordinates of the minimum pressure of the profiles are now known, the TLV trajectory line is found through linear fitting. The same procedure is repeated for finding the shock trajectory line, except that the axial coordinate of the minimum pressure is replaced by the axial coordinate of half the pressure rise across the shock.

Figure 7 shows the approximated trajectory lines and the intersection points of the shock and TLV trajectories for all operating points. The slope of the shock and TLV trajectory line are shown as angles, $\chi$ and $\xi$ in Fig. 8a. Both angles are measured from the axial direction. The angles are positive when measured in the direction of rotation. It shows that across all operating points, the TLV trajectory change is relatively small as compared to the shock trajectory. The slope of the shock trajectory line is shown to increase for about $20^{\circ}$ between operating points 4 and 10. This can be confirmed by examining the casing static pressure contour for operating points 4 and 8 as shown in Fig.6.
At operating point 8, the shock detaches from the blade and is inclined at a larger angle as compared to operating point 4 . The change of the shock trajectory as the compressor approaches stall causes the shock-TLV interaction location to move upstream. The normalised axial location $\left(\tilde{z}_{t}\right)$ of the intersection points between the shock and TLV are shown in Fig. 8b. For the last stable operating point (point 10), the shock-TLV interaction is located at about $15 \%$ of $c_{a x, t}$ aft of tip leading edge. As the compressor moves from ND to NS, the shock-TLV interaction travels upstream by approximately $10 \%$ of $c_{a x, t}$.

\section{Quantification of blockage}

The effect of the upstream movement of the shock-TLV interaction location on the blockage is investigated by quantifying the near casing blockage. The quantification of the near casing blockage is based on a qualitative mass flow overshoot method [7]. This method is adapted so that the blockage cells can be quantitatively evaluated. Blockage cells for an axial plane can be identified by sorting and summing the mass flow rate of each cell in a descending order. If negative mass flow (flow reversal) exists on that plane, summation of all cells with positive mass flow must overshoot the inlet mass flow value. The summation is stopped once the value overshoots the inlet mass flow rate. Remaining grid cells that are not summed are identified as 'blocked' and assigned a blockage index value of $\psi=1$. Grid cells that are not 'blocked' are assigned a value of $\psi=0$. The blockage index, $\psi$, is therefore used as a tool to identify 'blocked' cells in a plane.

Figure 9 shows the distribution of the non-dimensional blockage $\left(\Psi_{m}\right)$ for design and part speed for the top $20 \%$ span. The distribution of $\Psi_{m}$, as defined in Eq. 1, is calculated by extracting data for about 230 axial planes across the blade domain.

$$
\Psi_{m}=\frac{\sum_{i=1}^{N}|\psi(i) m(i)|}{\sum_{i=1}^{N} m(i)}
$$

$\Psi_{m}$ is the normalised absolute sum of the 'blocked' cell mass flow rate. The axial distribution of $\Psi_{m}$ evaluated for the top $20 \%$ span is plotted for selected operating points as shown in Fig. 3 to see the development of blockage as the compressor approaches stall. At design speed, as shown in Fig. 9a, the location of the peak blockage is seen to move further upstream towards the LE as the compressor approaches stall. The upstream movement of the peak blockage location is accompanied by an increase of the peak blockage value. This is consistent with the upstream movement of the shock-TLV interaction as mentioned earlier. Looking back at Fig. 8b, the shock-TLV interaction location is found to happen slightly upstream of the peak blockage location. For example, at Point 10, the shock-TLV interaction location is found to occur at about $15 \%$ of $c_{a x, t}$ whilst the peak blockage occurs at about $20 \%$ of $c_{a x, t}$. This shows that the blockage is gradually built-up before it peaks slightly 

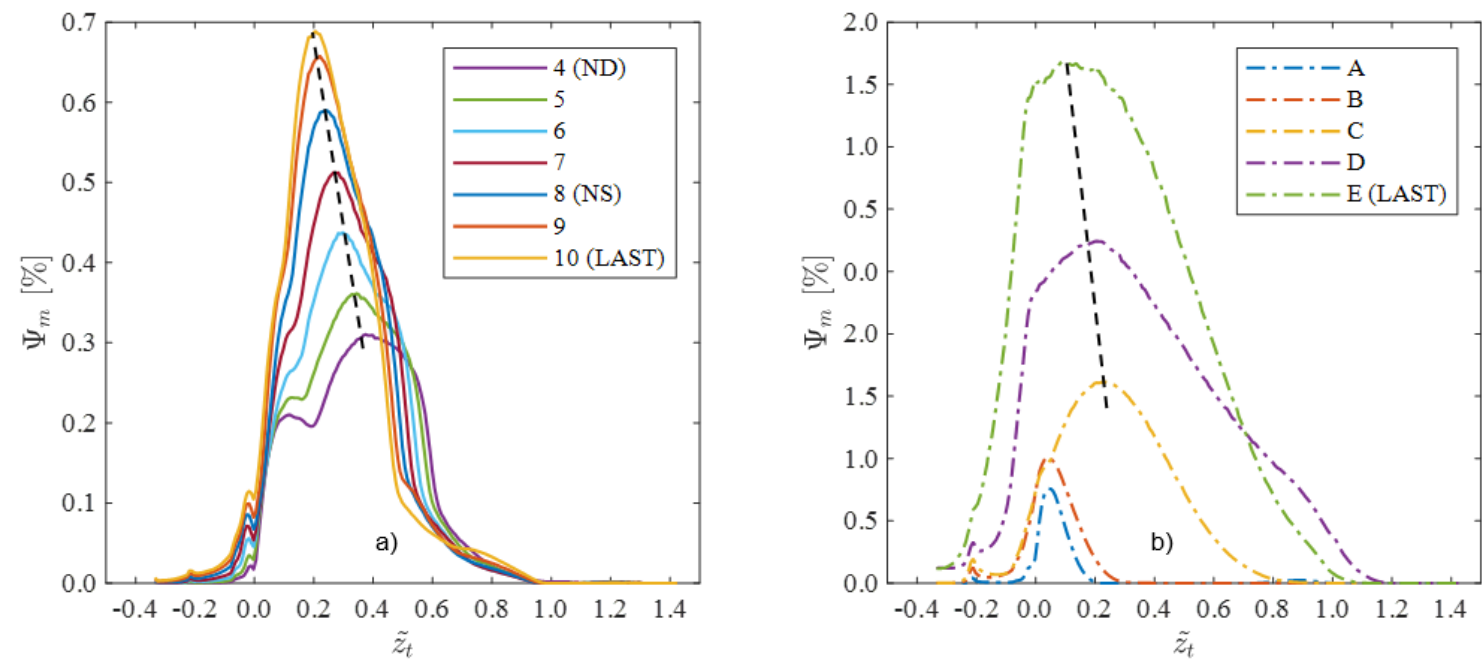

FIGURE 9. Non-dimensional mass flow blockage, $\Psi_{m}$, axial distribution over the top $20 \%$ span for a) design and b) part speed. The black dashed line shows the movement of the peak blockage as the compressor approaches stall.

downstream of the shock-TLV interaction location. The increase of the blockage peak shows that the strength of the shock-TLV interaction is higher as the compressor approaches stall. When the shock detaches from the blade as shown in Fig.6, the shock angle increases so that it interacts more with the upstream part of the TLV causing more blockage.

At part speed conditions as shown in Fig. 9b, the upstream movement of the peak blockage location is not as pronounced as the high-speed case due to the absence of the shock. Comparing the $\Psi_{m}$ distribution for design and part speed at the last stable operating point (Point 10 and E), the shape of the part-speed blockage distribution shows a higher and broader peak than the high-speed case. It is possible that due to the shock-TLV interaction, blockage for high speed is more confined to a smaller region as compared to the part-speed case where the shock is not present. As mentioned earlier, the intent of simulating the compressor at part-speed is only for comparing the blockage characteristics with the design speed case when the shock is present. Explaining the blockage at part-speed is not within the scope of this paper hence will not be presented here.

\section{GROOVED CASING RESULTS AND DISCUSSION}

By directly linking the characteristics of the blockage distribution with the physics of stall, a design optimisation method to obtain a single casing groove for SMI is attempted. This approach couples a surrogate model to a multi-objective evolutionary algorithm optimisation routine. The surrogate model is based on a supervised learning decision tree algorithm that uses training data from a space-filling design sampling method. The design of the casing groove is parameterised by four variables as shown in the inset of Fig. 1. Parameters $z^{\prime}, w^{\prime}, H$ and $\alpha$ are the normalised groove axial position, upper width, height and the upper internal angle, respectively. All parameters except $\alpha$ are normalised by the $c_{a x, t}$. The surrogate-based optimisation is performed at point 8 in Figure 3a which represents the near stall condition at design speed. The objectives of the optimiser algorithm are to search for a groove design that minimises the blockage at about $20 \%$ of $c_{a x, t}$ with the least efficiency penalty. The peak blockage axial location is chosen because intuitively this blockage is the reason for the onset of stall. The optimal solution of the casing groove design (S1) is shown in Tab. 2. Further details regarding the design optimisation procedure is explained in [16]. The SMI $(\Delta \zeta)$ for grooved casing is calculated using Eq. 2 as shown below.

$$
\Delta \zeta=\zeta_{G C}-\zeta_{S C} ; \quad \zeta=1-\frac{\pi_{4} m_{10}}{\pi_{10} m_{4}}
$$

Here, $\Delta \zeta$ is calculated by finding the difference between the stall margin of the grooved, $\zeta_{G C}$ and smooth casing, $\zeta_{S C}$. The stall margin, $\zeta$, is calculated using the $\pi$ and $m$ values from operating points 4 (ND) and 10 (last stable operating point).

TABLE 2. Design specification and performance of each groove casing configuration

\begin{tabular}{llllll}
\hline & $z^{\prime}$ & $w^{\prime}$ & $H / w^{\prime}$ & $\alpha^{\circ}$ & $\Delta \zeta[\%]$ \\
\hline S0 & 0.08 & 0.054 & 0.89 & 92 & 1.3 \\
S1 (optimised) & 0.169 & 0.054 & 0.89 & 92 & 0.6 \\
S2 & 0.26 & 0.054 & 0.89 & 92 & 0.13 \\
M1 (S0+S1) & & & & & 1.2 \\
M2 (S1+S2) & & & & & 0.5 \\
\hline
\end{tabular}



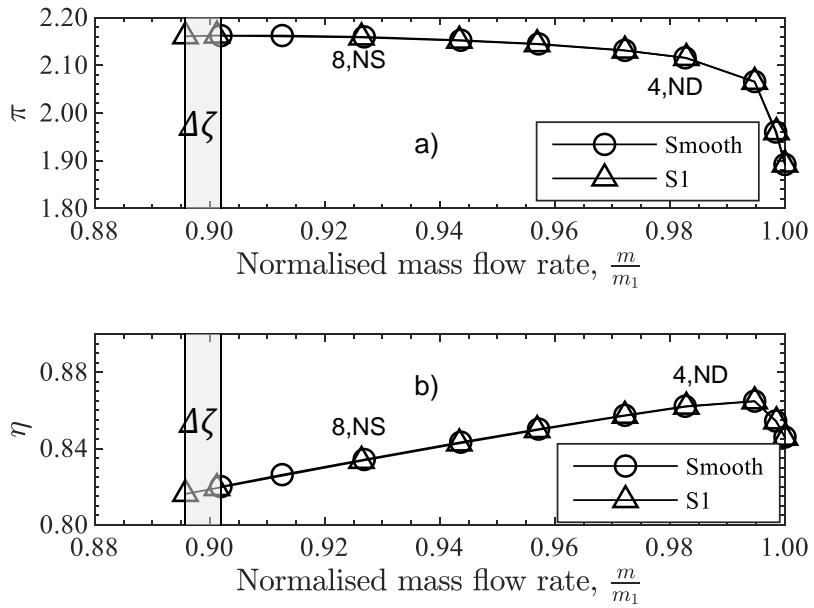

FIGURE 10. Comparison of the performance curve between smooth and optimised casing groove, S1. a) $\pi$ and b) $\eta$.

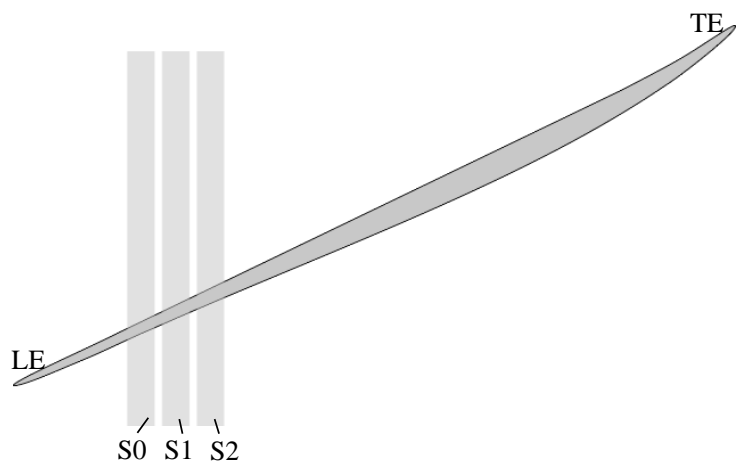

FIGURE 11. Relative position of the single casing grooves
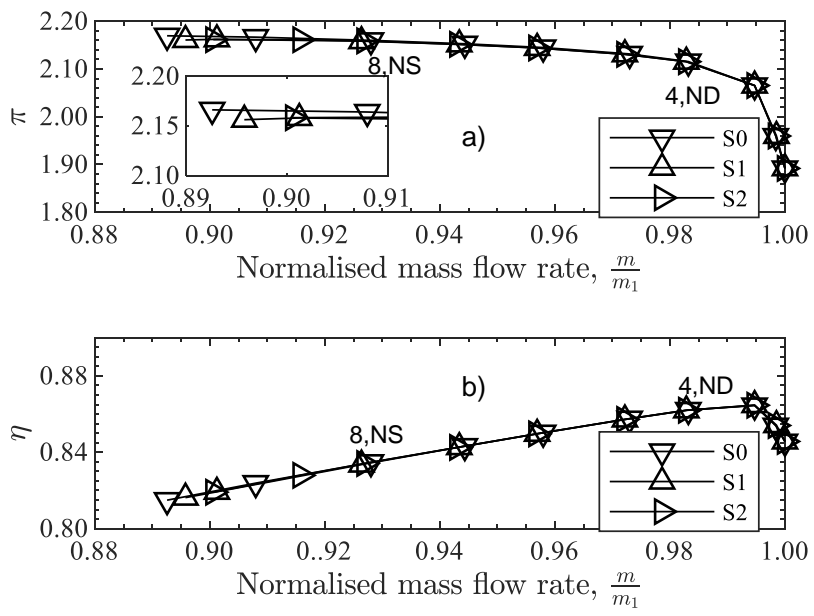

FIGURE 12. Performance curves of the single casing grooves. a) $\pi$ and b) $\eta$.

\section{Single groove}

Figure 10 shows the performance curve for the optimised casing groove design, S1, compared against the smooth casing. The stall point is found using the same convergence criteria used for the smooth casing. The stall margin improvement $(\Delta \zeta)$ for the optimised casing groove is about $0.6 \%$. The effect of groove S1 on $\eta$ is shown in Fig. 10b). It can be seen that groove S1 has negligible effect on $\eta$ across all operating points as compared to the smooth casing ( $\Delta \eta$ is within $0.1 \%$ at all operating points). Next, the sensitivity of the $\Delta \zeta$ to the groove axial location is investigated by moving the axial location of the groove geometry used in S1. The groove is moved upstream and downstream of its original position as in design $\mathrm{S} 1$ resulting in design S0 and $\mathrm{S} 2$, respectively. Figure 11 shows the axial location of S0 and S2 with respect to S1. Details regarding S0 and S2 are found in Tab. 2. Figure 12 shows the performance curve of S0, S1 and S2. The $\Delta \zeta$ for S0 and S2 are $1.3 \%$ and $0.13 \%$, respectively. It is found that the performance of the groove is better when located upstream of the peak blockage location as $\Delta \zeta$ of S0 is twice that of the optimised groove. On the other hand, the performance of $\mathrm{S} 2$ is worse than S1. Moving the groove downstream of the peak blockage location reduces the $\Delta \zeta$ to $0.13 \%$. However, it is found that varying the axial location of the groove $\mathrm{S} 1$ show no significant effect on $\eta$ where the $\eta$ curve for S0, S1 and S2 as shown in Fig. 12b) are overlapping. A similar trend is also reported in [7]. It was suggested that the casing groove attenuated the TLV which results in negligible efficiency penalty.
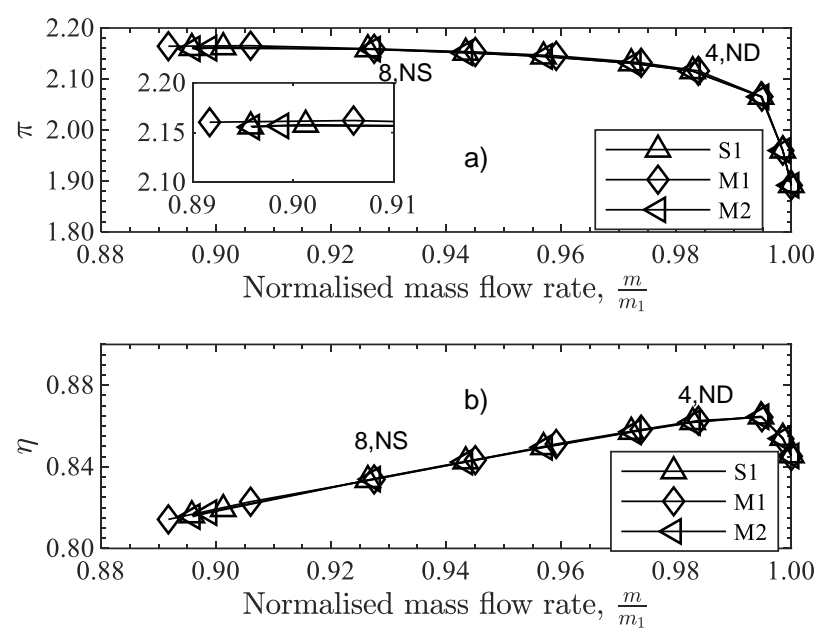

FIGURE 13. Comparison of the performance curve between $\mathrm{S} 1$ and the multiple casing grooves. a) $\pi$ and b) $\eta$.

\section{Multiple grooves}

The effect of a multi-groove configuration on the $\Delta \zeta$ is investigated by combining the single grooves. The first configuration, $\mathrm{M} 1$, combines $\mathrm{S} 0$ and $\mathrm{S} 1$ while $\mathrm{M} 2$ is a combination of S1 and S2. The performance curve of M1 and M2 are compared against S1 and are shown in Fig. 13. The $\Delta \zeta$ of each case is summarised in Tab. 2. It is found that having a multi-groove configuration does not result in a linear increase of the $\Delta \zeta$ as what have been found in [9]. However, since different rotors have distinct blockage features, it is difficult to expect any similarity between these findings. The $\Delta \zeta$ of M1 and M2 are comparable to the $\Delta \zeta$ of the upstream single groove in each multi-groove configuration. For example, the $\Delta \zeta$ of M1 is similar 
to S0. This is also the case with M2 where the $\Delta \zeta$ of it is similar to $\mathrm{S} 1$. In terms of $\eta, \mathrm{M} 1$ and $\mathrm{M} 2$ show comparable value with $\mathrm{S} 1$ across all operating points.

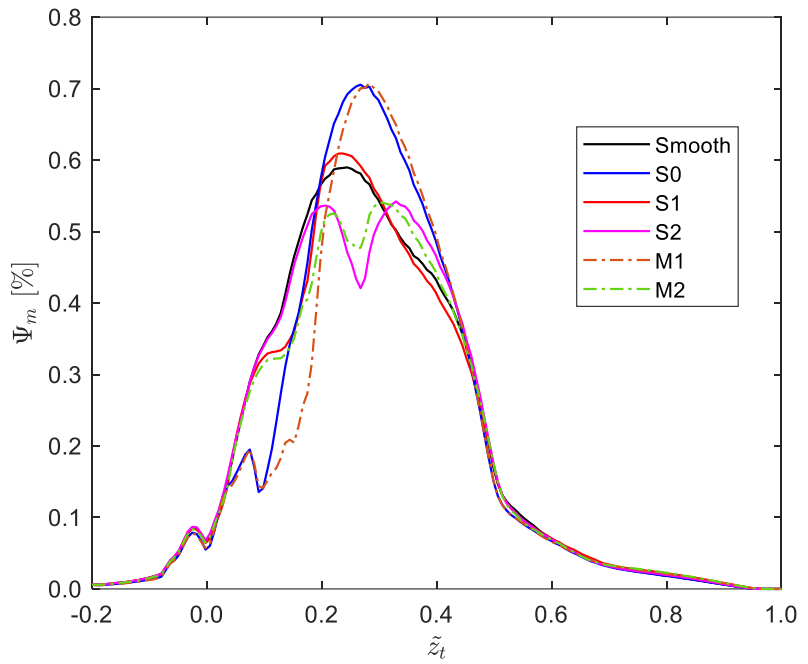

FIGURE 14. Comparison of the $\Psi_{m}$ axial distribution for all grooved casing cases at operating point 8

\section{Relationship between blockage reduction and SMI}

Figure 14 shows the effect of the groove on the $\Psi_{m}$ distribution at operating point 8 . Clearly, the location of the groove plays an important role on where the blockage is modified. Compared with the smooth casing, S0 and S1 have considerably lower blockage upstream of the blockage peak. S2 on the other hand reduces the peak blockage itself. Since S0 has the best $\Delta \zeta$ as compared to other cases, it can be said that removing front part of the peak blockage is more beneficial than reducing the peak blockage itself. S0 is located between $8 \%$ and $14 \%$ of the $c_{a x, t}$ which is upstream of the peak blockage location
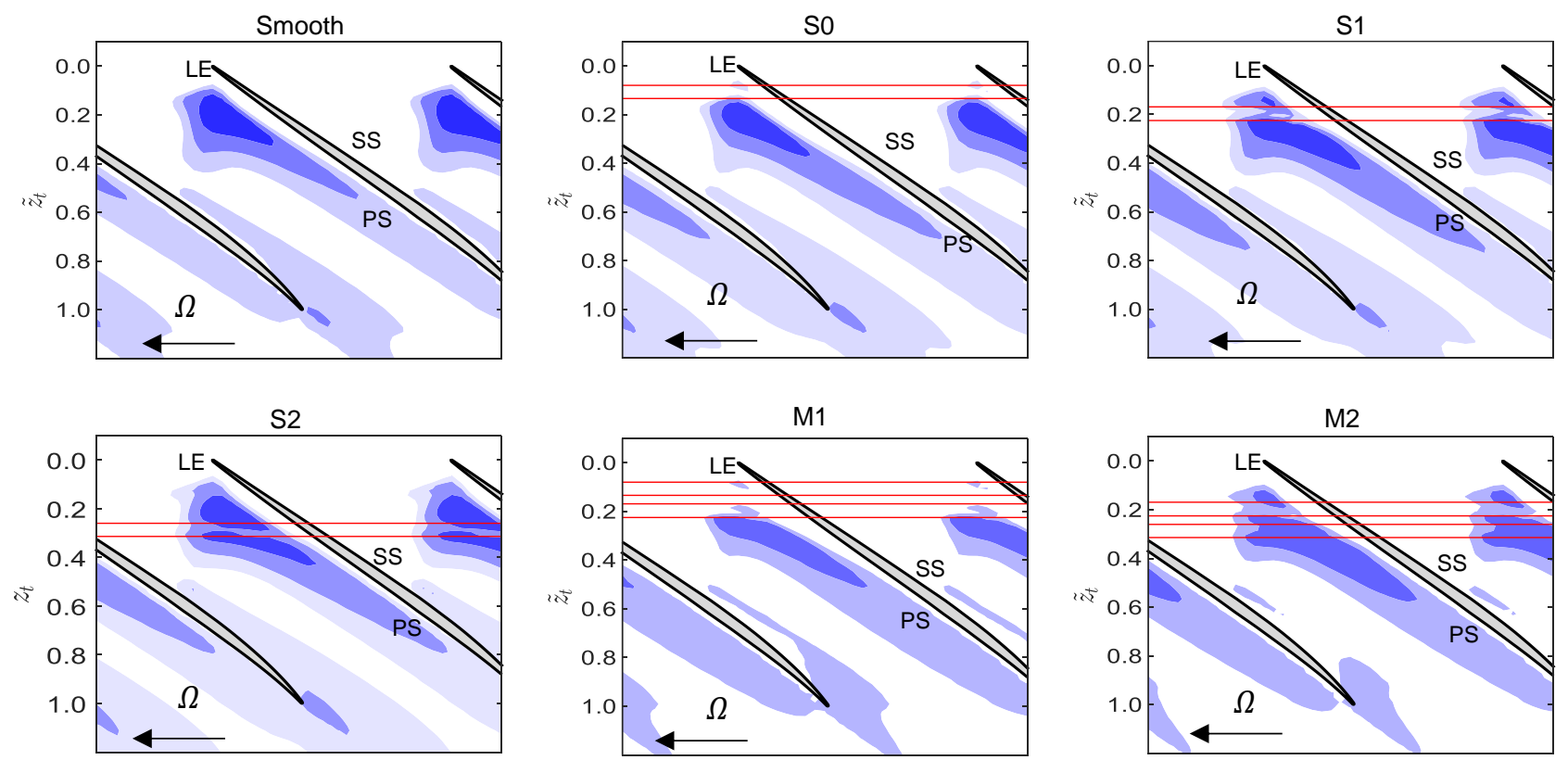

(about $20 \% c_{a x, t}$ ) and also the shock-TLV intersection location (about $15 \% c_{a x, t}$ ). This means that S0 may have affected the origin of the blockage itself, which from the previous discussion, is thought to be the shock-TLV interaction. The blockage distribution for M1 and M2 follows the same trend of that of S0 and $\mathrm{S} 1$ respectively with slightly more reduction in the blockage seen. However, the increase of the blockage reduction is not reflected on the improvement of the $\Delta \zeta$.

The effect of the groove on the blockage can be further analysed by plotting the Mach number contour for operating point 8 at the mid-tip gap region as shown in Fig 15. Low Mach number regions are coloured in darker shades of blue to show the blockage. For the smooth casing, the blockage region is located closer to blade pressure side and extends slightly downstream aft of the tip leading edge. As what has been discussed earlier, the distribution of the blockage region is affected by the groove when compared to the smooth casing. This is reflected either as a reduction of the size or a decreased intensity of the low Mach number region when the groove is present. The front part of the blockage region of $\mathrm{S} 0$ is attenuated by the groove as compared to S1 and S2. Removal of this upstream part of the blockage allows the incoming flow to enter the blade passage with reduced resistance which results in an increase in the $\zeta$. For S2, the groove only attenuates the aft part of the blockage while the front part of the blockage still exists. The upstream part of the blockage prevents incoming flow from entering the blade passage easily and this causes a reduction in any gain in SMI $(\Delta \zeta)$. Therefore, this clearly shows that the $\Delta \zeta$ gained by each configuration depends on the location where the blockage is removed. The Mach number contours for M1 and M2 follow the same trend as S0 and S1 in terms of the shape of the blockage region.

FIGURE 15. Mach number contour plots at a constant span plane inside the tip gap region at operating point 8 

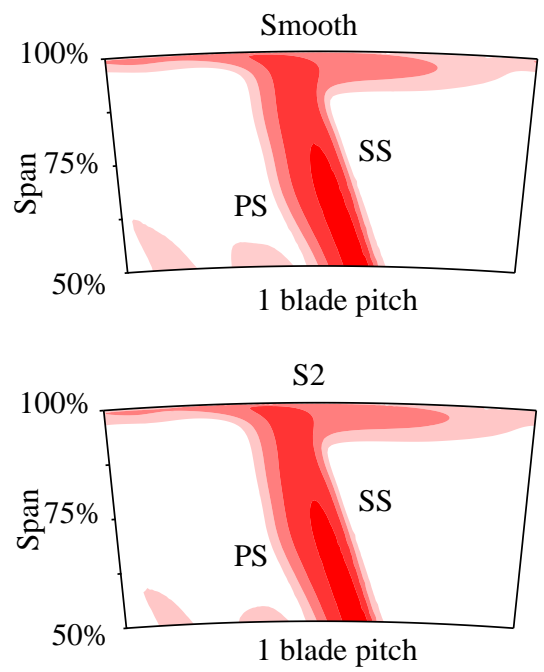
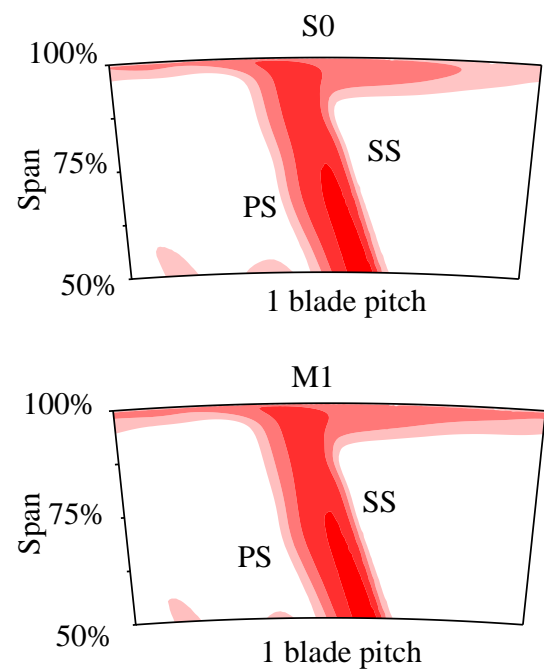
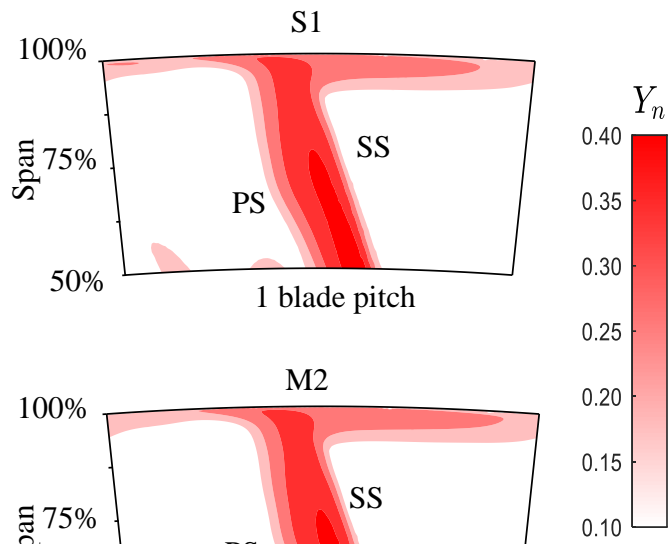

FIGURE 16. Contours of $Y_{n}$, aft of the blade at $30 \%$ of the $c_{a x, t}$ downstream of the tip TE at operating point 8 .

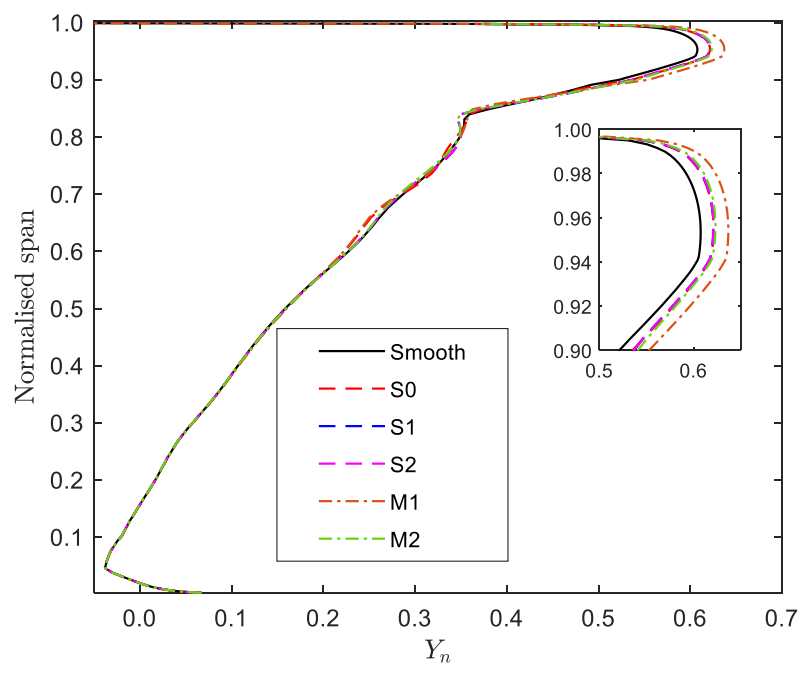

Figure 17. Pitchwise mass-averaged values of $Y_{n}$ aft of the blade

\section{Near casing downstream losses}

Figure 16 shows the contours of the total pressure loss coefficient, $Y_{n}$, at operating point 8 for all the cases. $Y_{n}$ is defined using Eq. 3 where $P_{02, r e l}$ is the relative total pressure at $30 \%$ of the $c_{a x, t}$ downstream of the tip trailing edge (TE). $P_{01, r e l}$ and $P_{1}$ are the inlet relative total pressure and static pressure, respectively.

$$
Y_{n}=\frac{P_{02, r e l}-P_{01, r e l}}{P_{01, r e l}-P_{1}}
$$

For clarity, only the top $50 \%$ span region is shown. From visual inspection, it can be seen that the groove increases the circumferential extent of loss at the near casing region as compared to the smooth casing. The near casing loss for the single grooved cases S0, S1 and S2 are comparable with each other. Multi-groove configurations on the other hand show larger loss region near the casing. Figure 17 shows the pitchwise massaverage of $Y_{n}$ at the same location as where the contours are plotted. The spanwise $Y_{n}$ distribution for the bottom $50 \%$ span region show no change as compared to the smooth casing. By zooming into the top $10 \%$ as shown in the inset of Fig 17, it can be clearly seen that adding more grooves result in additional losses as M1 and M2 show a higher loss compared to the single grooved cases. This may explain why M1 and M2 configurations show a slight reduction in $\Delta \zeta$ as compared to the single grooved cases $\mathrm{S} 0$ and $\mathrm{S} 1$ although the multi-groove configurations show a larger overall blockage reduction as shown in Fig. 14. The momentum transfer of flow between the groove and tip gap region and the increased wetted area are thought to be responsible for the generation of losses. However, the near casing losses due to casing grooves calculated at operating point 8 show negligible effect on $\eta$ as shown in Fig. 10, 12 and 13.

\section{CONCLUSIONS}

A 3D steady RANS simulation has been a performed to study the effects of casing grooves on the SMI of a transonic compressor rotor. The conclusions of this study can be summarised as the following:

1. The shock-TLV interaction is found to be the main source of the near casing blockage. The location of the shock-TLV interaction is found by intersecting the trajectories of shock and TLV. As the compressor approaches stall, the location of the intersection point is found to move in the upstream direction. At the last stable operating point, the shock-TLV interaction is found to occur at about $15 \%$ of $c_{a x, t}$.

2. The near casing blockage is quantified using a nondimensional blockage parameter, $\Psi_{m}$. The distribution of 
$\Psi_{m}$ is plotted for several operating points at design and part speed conditions. At design speed, the peak blockage location is found to follow the same trend of the upstream movement of the shock-TLV intersection point as the compressor approaches stall. This trend is not pronounced at part-speed conditions as the blockage in part-speed conditions is not influenced by the shock.

3. Based on the information obtained from the smooth casing $\Psi_{m}$ distribution, a single casing groove design is generated through a design optimisation approach. The optimal casing groove, $\mathrm{S} 1$, is found to improve the $\zeta$ by $0.6 \%$. The axial position of this groove is varied to obtain two more single groove configurations, $\mathrm{S} 0$ and $\mathrm{S} 2$. The grooves for S0 and S2 are respectively located upstream and downstream of the peak $\Psi_{m}$ location for the smooth casing. From this parametric study, S0 is found to have the best SMI as compared to S1 and S2. The $\Delta \zeta$ of S0 is found to be about twice the $\Delta \zeta$ of S1. S0 which is located upstream of the shock-TLV interaction location is found to remove the blockage, at this location, which prevents flow from entering the blade passage. This in return results in the best gain in $\Delta \zeta$. Adding the single grooves together to form multi-groove configurations do not show any significant change to the $\Delta \zeta$. It was found that the $\Delta \zeta$ of a multi-groove configuration follows the same trend as the upstream groove in each configuration. Although, mass-averaged pressure loss profiles show marginally higher losses near the casing for multi-groove configurations, the efficiency maps suggest that there is negligible efficiency variation $(<$ $0.1 \%$ ) across all cases presented.

\section{ACKNOWLEDGEMENTS}

The first author would like to thank the Ministry of Education, Malaysia and Universiti Sains Malaysia for providing the financial support towards his research studentship.

\section{REFERENCES}

[1] Suder, K. L., and Celestina, M. L., 1996. "Experimental and Computational Investigation of the Tip Clearance Flow in a Transonic Axial Compressor Rotor". Journal of Turbomachinery, 118(2).

[2] Brandstetter, C., Jüngst, M., and Schiffer, H.-P., 2018. "Measurements of Radial Vortices, Spill Forward, and Vortex Breakdown in a Transonic Compressor". Journal of Turbomachinery, 140(6).

[3] Yamada, K., Kikuta, H., Iwakiri, K., Furukawa, M., and Gunjishima, S., 2012. "An Explanation for Flow Features of Spike Type Stall Inception in an Axial Compressor Rotor". Journal of Turbomachinery, 135(2).

[4] Pullan, G., Young, A. M., Day, I. J., Greitzer, E. M., and Spakovszky, Z. S., 2015. "Origins and Structure of Spike-Type Rotating Stall". Journal of Turbomachinery, 137(5).

[5] Bailey, E. E., 1972. "Effect of Grooved Casing Treatment on the Flow Range Capability of a Single-stage axialflow compressor". NASA Technical Report (NASA-TM-
X 2459, E-6560), p. 17.

[6] Muller, M. W., Schiffer, H.-P., and Hah, C., 2007. "Effect of Circumferential Grooves on the Aerodynamic Performance of an Axial Single-Stage Transonic Compressor". In Proceedings of the ASME Turbo Expo 2007: Power for Land, Sea, and Air. Volume 6: Turbo Expo 2007, Parts A and B., pp. 115-124.

[7] Sakuma, Y., Watanabe, T., Himeno, T., Kato, D., Murooka, T., and Shuto, Y., 2013. "Numerical Analysis of Flow in a Transonic Compressor with a Single Circumferential Casing Groove: Influence of Groove Location and Depth on Flow Instability". Journal of Turbomachinery, 136(3).

[8] Chen, H., Huang, X., Shi, K., Fu, S., Ross, M., Bennington, M. A., Cameron, J. D., Morris, S. C., McNulty, S., and Wadia,A, 2013. "A Computational Fluid Dynamics Study of Circumferential Groove Casing Treatment in a Transonic Axial Compressor". Journal of Turbomachinery, 136(3).

[9] Ross, M. H., Cameron, J. D., Morris, S. C., Chen, H., and Shi, K., 2017. "Axial Compressor Stall, Circumferential Groove Casing Treatment, and the Tip-Clearance Momentum Flux". Journal of Propulsion and Power, 34(1), pp. 146-152.

[10] Suder, K. L., 1996. "Experimental Investigation of the Flow Field in a Transonic, Axial Flow Compressor with Respect to the Development of Blockage and Loss". PhD thesis, Case Western Reserve University, USA.

[11] Denton, J. D., 1997. "Lessons from Rotor 37”. Journal of Thermal Science, 6(1), pp. 1-13.

[12] ANSYS CFX 17.1 Documentation.

[13] Dunham, J., 1998. CFD Validation for Propulsion System. Tech. rep., Advisory Group for Aerospace Research \& Development (AGARD), Neuilly-Sur-Seine, France.

[14] Bruna, D., and Turner, M. G., 2013. "Isothermal Boundary Condition at Casing Applied to the Rotor 37 Transonic Axial Flow Compressor". Journal of Turbomachinery, 135(3).

[15] Furukawa, M., Inoue, M., Saiki, K., and Yamada, K., 1999. "The Role of Tip Leakage Vortex Breakdown in Compressor Rotor Aerodynamics". Journal of Turbomachinery, 121(3).

[16] Mustaffa, A. F., Kanjirakkad, V., 2019. "Design Optimisation of Circumferential Casing Groove for Stall Margin Improvement in a Transonic Compressor Rotor". Proceedings of the 54th $3 \mathrm{AF}$ International Conference on Applied Aerodynamics, p. 10. 